\title{
LA COUPURE \\ DE L'ESTUAIRE DE LA RANCE
}

\section{THE CLOSURE OF THE RANCE ESTUARY}

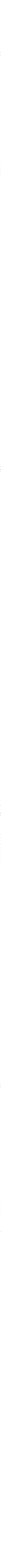

LA HOUILLE BLANCHE - No 7-1963 


\section{LA COUPURE DE L'ESTUAIRE DE LA RANCE}

\section{THE CLOSURE OF THE RANCE ESTUARY}

L'Electricité de France a entrepris la construction dans la vallée de la Rance de la première usine marémotrice du monde. Une étape particulièrement importante des travaux vient d'être franchie avec l'exécution du batardeau coupant en eau vive le débit de marée qui traverse l'estuaire. Les photographies que nous publions aujourd'hui illustrent les diverses phases de cette opération.

The first tidal power station in the world is now being built in the Rance river valley for Electricite de France. A particularly important stage in this work bas just been completed witb the construction of the cofferdam cutting off the tidal flow through the estuary. Photograpbs illustrating the various phases of this operation will be found in this issue.

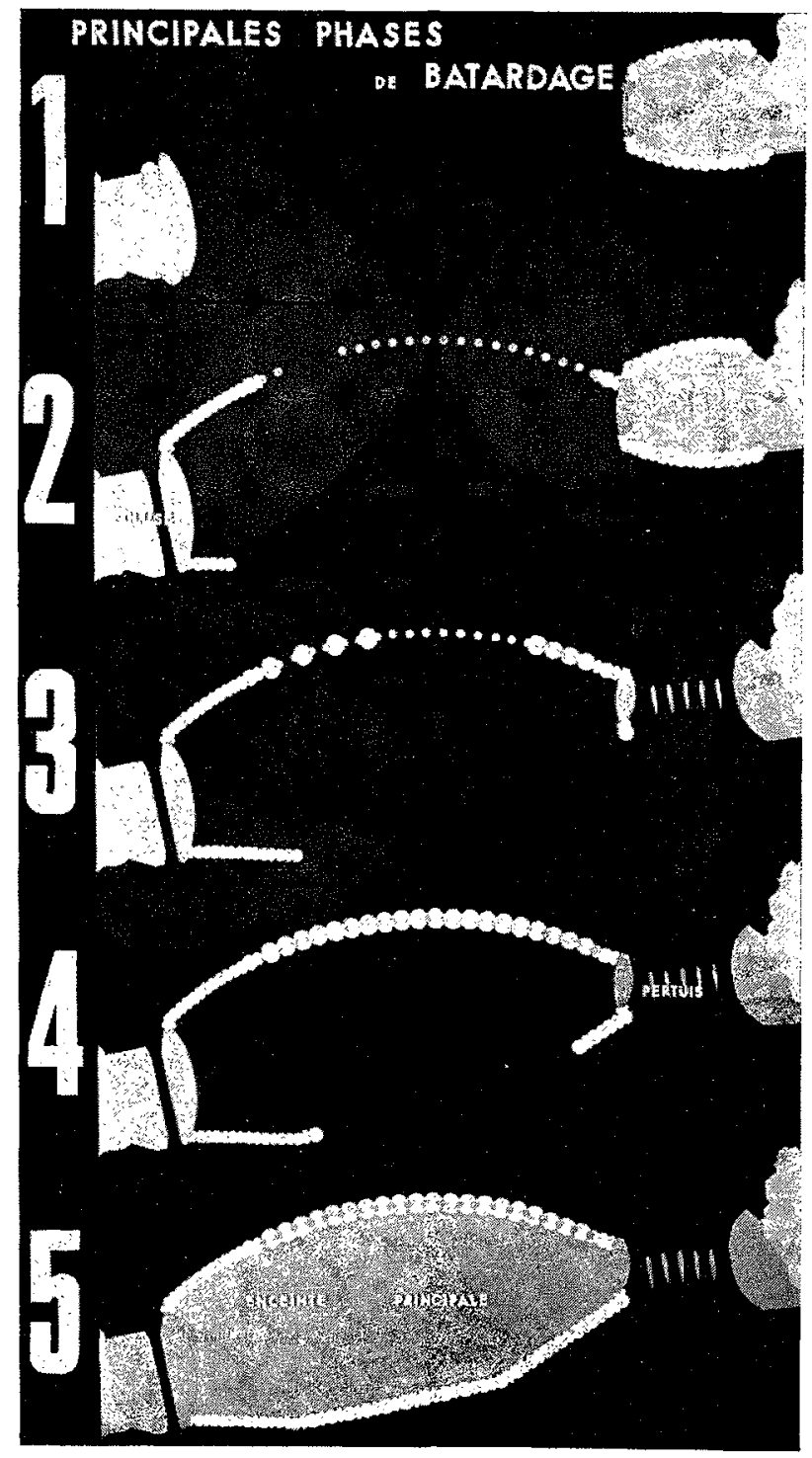

\begin{abstract}
$\mathrm{N}$ ristiques générales de l'aménagement de la Rance. L'estuaire, rappelons-le, est soumis à une marée qui peut atteindre $13,5 \mathrm{~m}$. Au cours d'une marée exceptionnelle, il emmagasine au flot et restitue au jusant un volume d'eau de l'ordre de 180 millions de $\mathrm{m}^{3}$, le débit instantané atteignant $18000 \mathrm{~m}^{3} / \mathrm{s}$. A l'emplacement choisi pour le barrage, la largeur de l'estuaire est d'environ $750 \mathrm{~m}$ et les fonds les plụs bas sont à $12 \mathrm{~m}$ all-dessous du zéro des cartes marines (à partir duquel nous domnerons les diverses cotes dans la suite de cet exposé). Ces fonds sont constitués par du rocher recouvert de sable, de galets ou de vase sur un mètre d'épaisseur en moyenne.
\end{abstract}

$\mathrm{L}$ E procédé de coupure a été décrit en détail dans notre Numéro Spécial consacré à la Rance (n" 2/1962, p. 117 à 129). Une description rapide des diverses phases de cette opération facilitera cependant l'intelligence des photographies qui suivent.

$\mathrm{P}$

REMIÉRE PHASE. - Dès l'origine des travaux, deux chantiers d'importance moyenne sont ouverts:

A la pointe de la Brebis, sur la rive gauche (a ganehe sur notre schéma), un mur batardeau en 
béton est construit «à la marée». Ce mur batardeau, qui par la suite est incorporé dans les ouvrages définitifs, permet de construire l'écluse. (longueur du sas $65 \mathrm{~m}$, largeur $13 \mathrm{~m}$, radier à la cote +2 ).

Sur la rive droite, partant de la pointe de la Briantais et s'appuyant sur l'îlot de Chalibert, une digue de précoupure en enrochements permet de réaliser à l'abri du courant une enceinte constituée par des gabions de palplanches de $19 \mathrm{~m}$ de diamètre remplis de sable et arasés au niveau +14. A l'intérieur de cette enceinte, la construction du barrage mobile est immédiatement entreprise (radier à la cote -4 , six passages de $15 \mathrm{~m}$ de large et $10 \mathrm{~m}$ de haut obstrués par des vannes-wagon).

EUXIÈME PHASE. - La réalisation de l'enceinte principale est commencée par la construction du batardeau Nord.

- partant de l'extrémité Nord de l'écluse et de l'îlot de Chalibert, les amorces de l'enceinte principale sont construites en gabions de palplanches de $19 \mathrm{~m}$ de diamètre;

- un caisson à air comprimé permettant de travailler sous l'eau prépare des fondations isolées pour l'échouage des caissons;

- 19 caissons cylindriques en béton, de $9 \mathrm{~m}$ de diamètre, sont préfabriqués dans une cale sèche du port de Saint-Malo, puis remorqués et échoués en les basculant sur les fondations préparées précédemment.

$\mathrm{T}$ ROISIĖME PHASE. - Dès l'achèvement du barrage mobile, le batardeau est démonté et les pertuis mis en service, afin de réduire l'obstruction créée dans la rivière par le batardeau Nord en construction.

Les intervalles entre caissons sont fermés à raison de un sur deux par des poutrelles provisoires, à l'abri desquelles sont construites des cellules de palplanches réunissant les caissons deux à deux, afin de créer des points d'appuis plus stäbles que ne l'étaient les seuls caissons. La dénivellation entre les deux côtés du bátardeau augmente avec l'obstruction de l'estuaire. Elle atteignait 2 mètres le 24 mai 1963 et 3 mètres le 24 juin.

Les intervalles restés libres entre les groupes de caissons sont à leur tour fermés par des poutrelles, puis par des cellules de palplanches jusqu'à la fermeture totale du batardeau Nord. Le 20 juillet 1963, les vannes étaient fermées, et la Rance définitivement isolée de la mer.

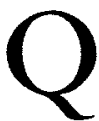
alors fermées et le plan d'eau dans l'estuaire est maintenu à la cote $+8,50$ pour permettre la navigation dans la partie amont de l'estuaire jusqu'à l'écluse du Chatelier.

L'achèvement du batardeau Sud, constitué uniquement de gabions de palplanches arasés à la cote +9 , se fait en eau morte.

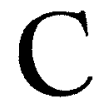
la construction de l'usine et de la digue peut être entreprise dès que l'assèchement de l'enceinte principale est terminée.

\section{L}

'USINE sera constituée par' une digue creuse en béton de $390 \mathrm{~m}$ de long et $53 \mathrm{~m}$ de large, arascée à la cote +15 et fondée à la cote -10 . Elle sera équipée de 24 groupes bulbe de $10 \mathrm{MW}$. L'accès se fera par un puits implanté sur la pointe de la Brebis et une galerie passant sous l'écluse.

La digue en enrochements qui reliera l'extrémité Est de l'usine à l'îlot de Chalibert, aura $175 \mathrm{~m}$ de long. Un noyau de béton assurera son étanchéité. A l'heure actuelle, l'assèchement est pratiquement achevé et les travaux de construction de l'usine et de la digue sont commencés. 


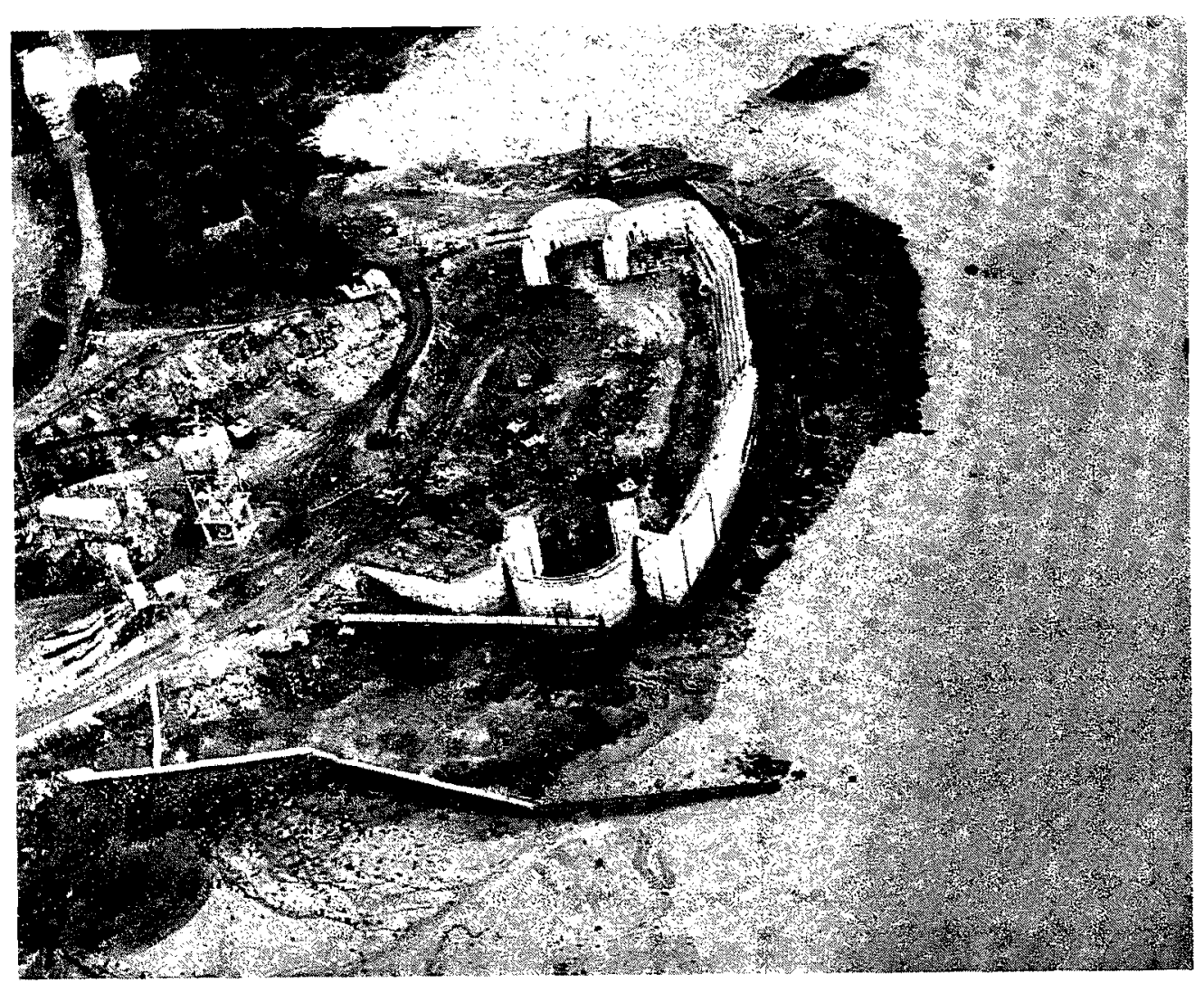

Le chantier de l'écluse. Construction du mur batardeau.

Work in progress on the lock cofferdam.

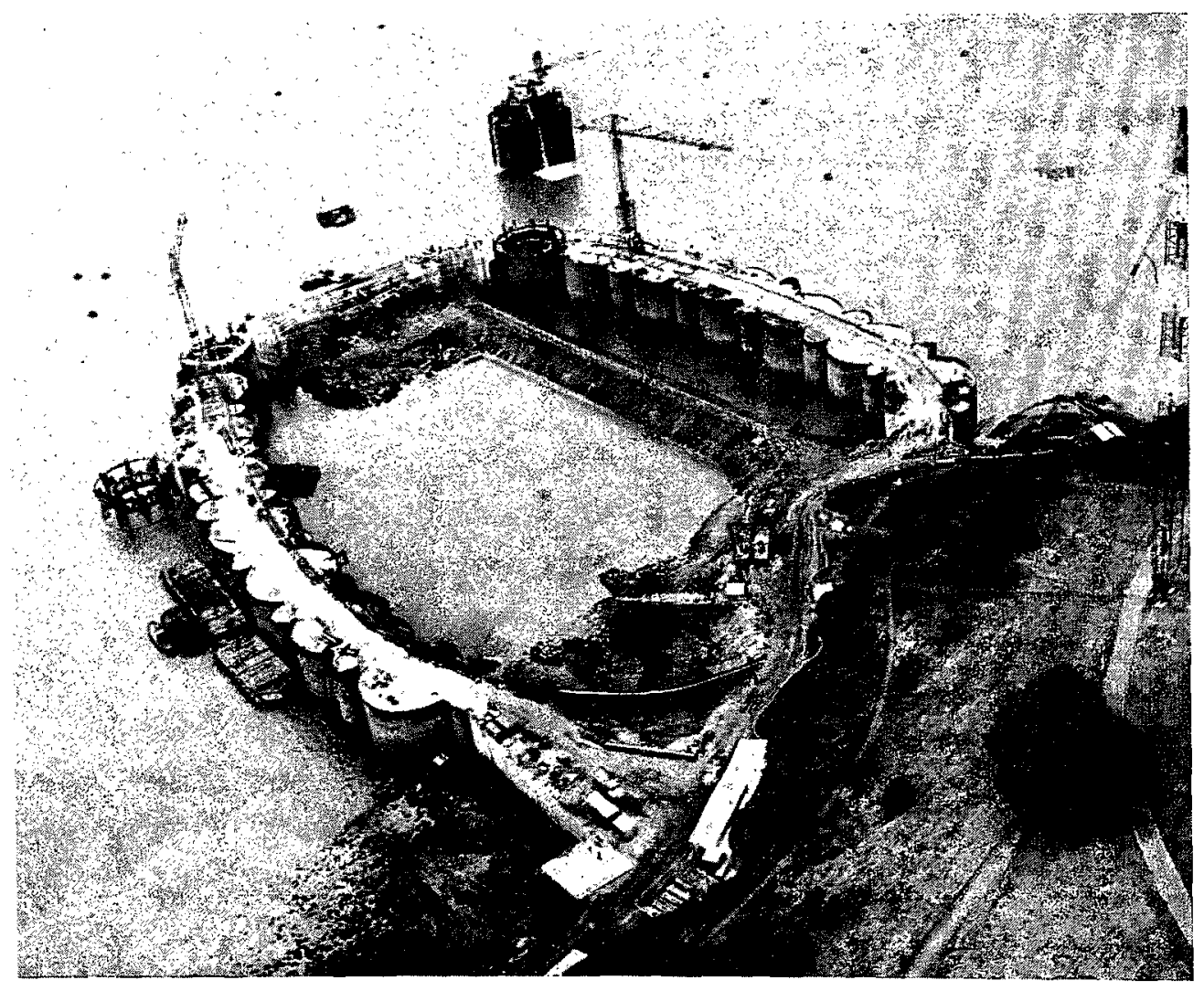

L'enceinte de gabions de palplanches destinée à la construction du barrage mobile. Lo digue de précoupure est encore en place.

The sheet piling cofferdam protecting the dam spillway section construction site, showing the original preliminary cutoff dyke still in position. 
Le caisson à air comprimé prépare la fondation destinée à l'échouage d'un caisson cylindrique en béton.

Use of a compressed air caisson

to prepare foundations

for a cylindrical concrete caisson.

Le caisson préfabriqué, de $9 \mathrm{~m}$ de diamètre, est remorqué jusqu’à son emplacement définitif.

A 30-foot diameter prefabricated concrete caisson being towed

to its final position.

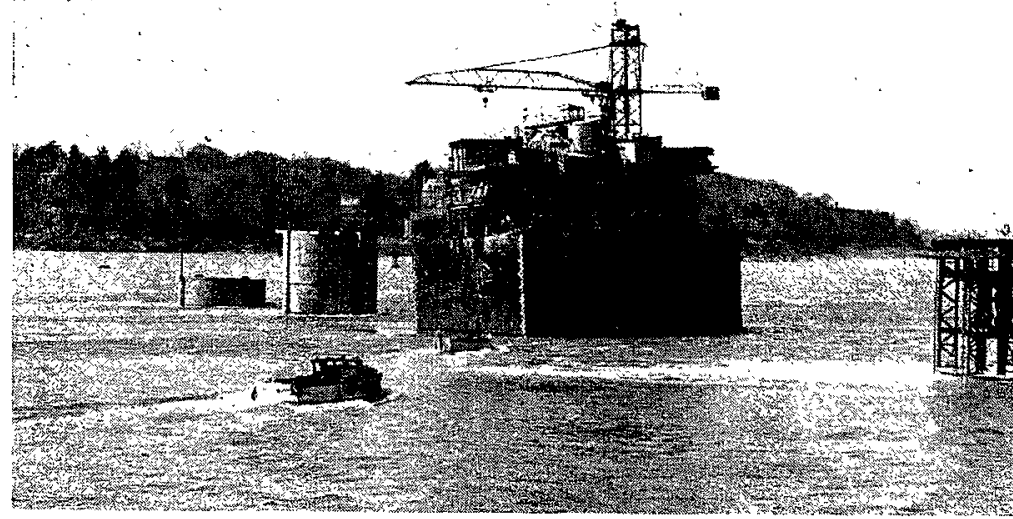

Basculement du caisson.

Tipping the caisson over.

Echouage du caisson sur son socle de béton.

Positioning the caisson on its concrete foundation
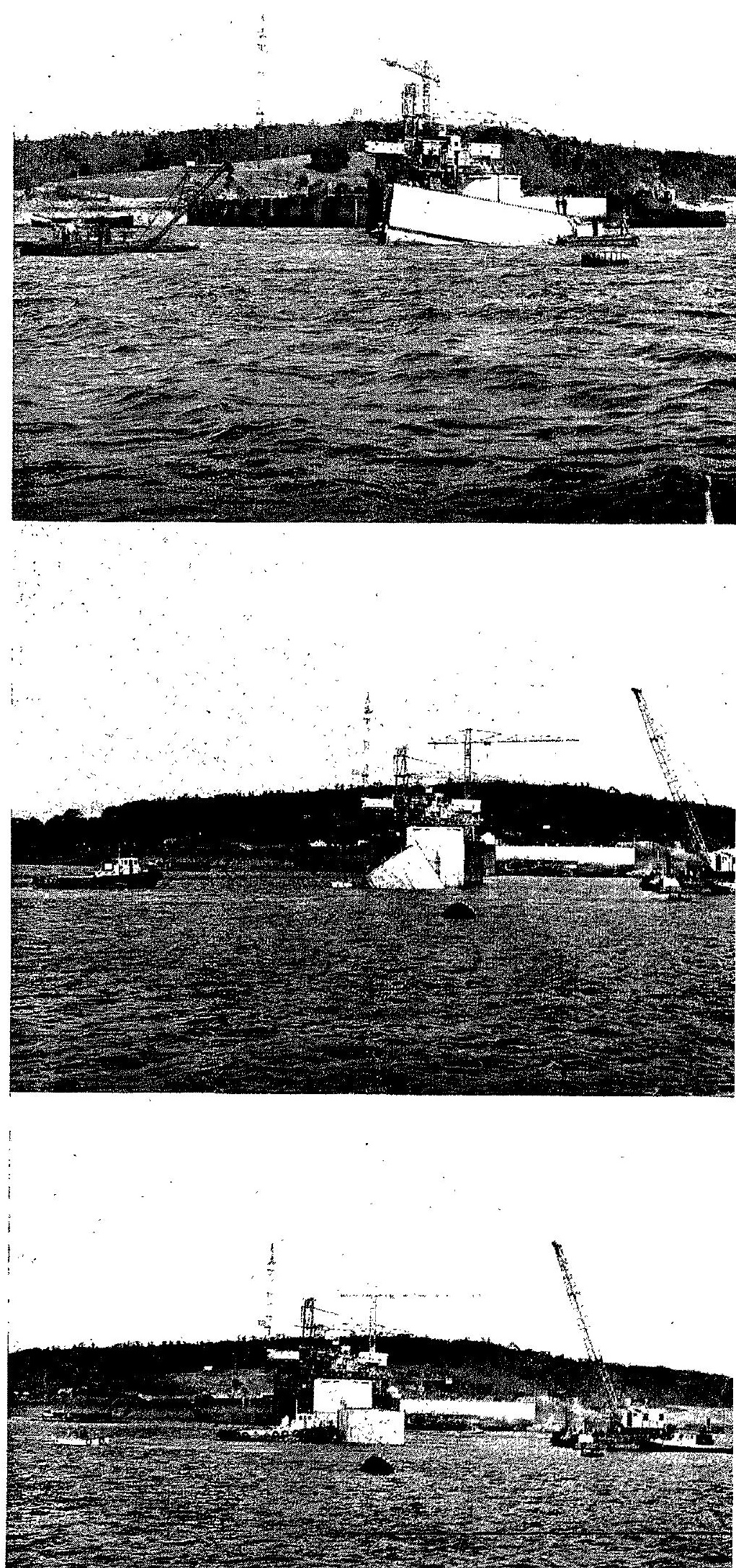


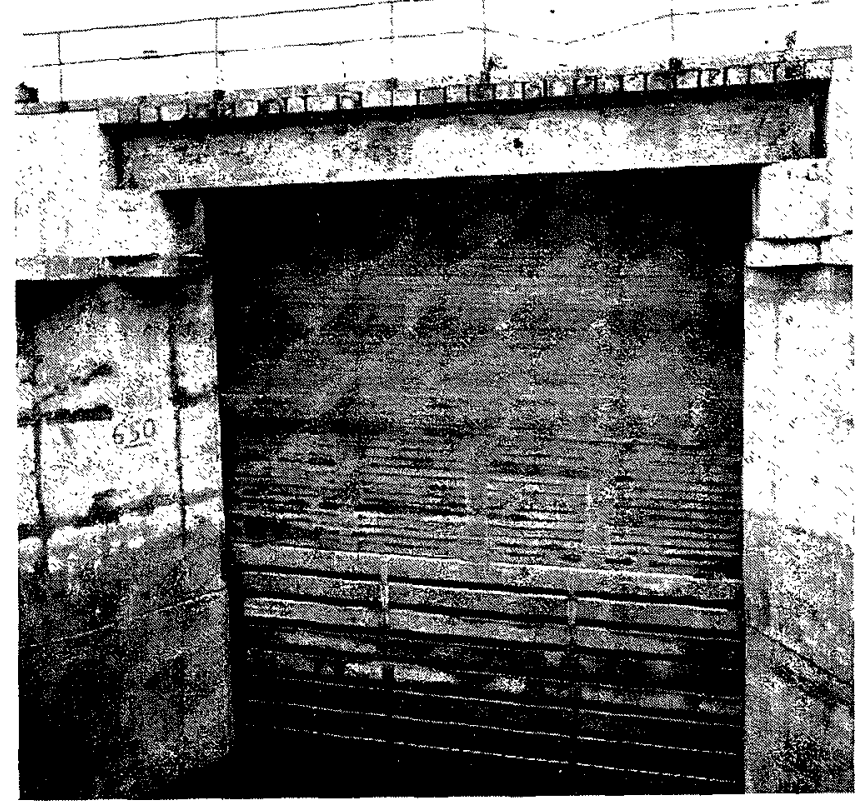

Fermeture au moyen de poutrelles provisoires de l'intervalle (11 mètres) entre deux caissons.

Temporary stop beams in position closing the 36-foot gap between two caissons.

Gabarit flottant extérieur pour la construction des cellules de palplanches.

Après accrochage sur les caissons de béton armé, les ballasts sont remplis d'eau.

A floating jig used in building the sheet piling cells.

Once it has been attached to the outside of the caisson its ballast tanks are filled with water.

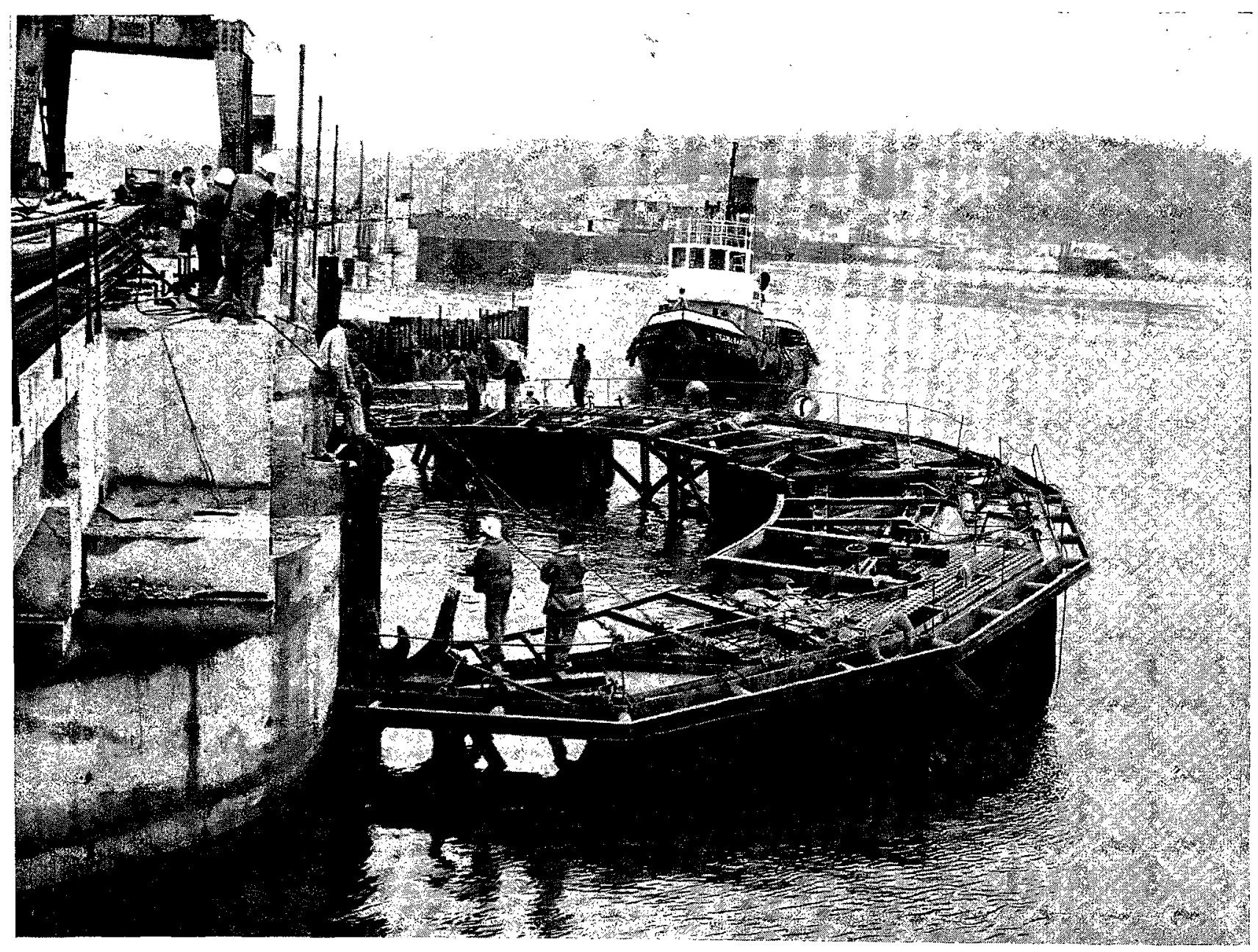






Courant de flot à travers le batardeau Nord en marée de coefficient 109 ( 25 mars 1963).

Les six vannes du barrage mobile sont ouvertes.

La dénivelée maximale à cette marée a été seulement de 0,8 mètre, alors que dans les mêmes conditions de marée, en février, elle avait atteint 1,5 mètre.

Flood tide through the north cofferdam on the 25th March 1963 (Tide coefficient 109) With all six weir gates open, the maximum difference in water levels across the dam was only about $2^{\prime \prime} 7^{\prime \prime}$, as compared to nearly 5 feet under the same conditions the month before.

Détail de l'écoulement le long d'un caisson de béton armé.

Close-up of the flow along a reinforced concrete caisson.

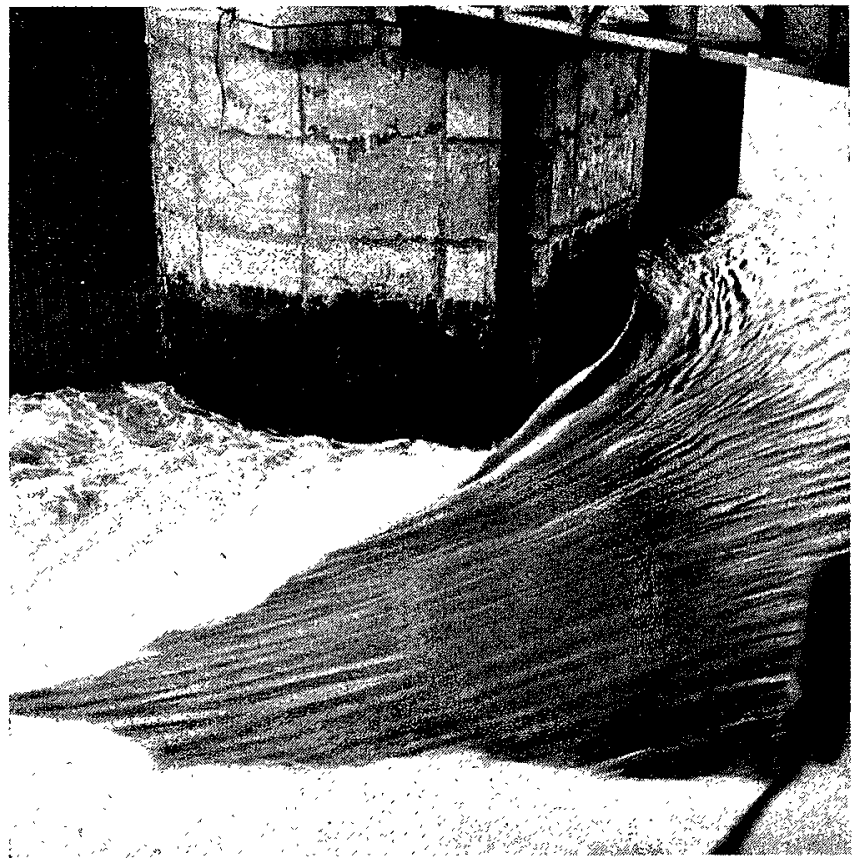




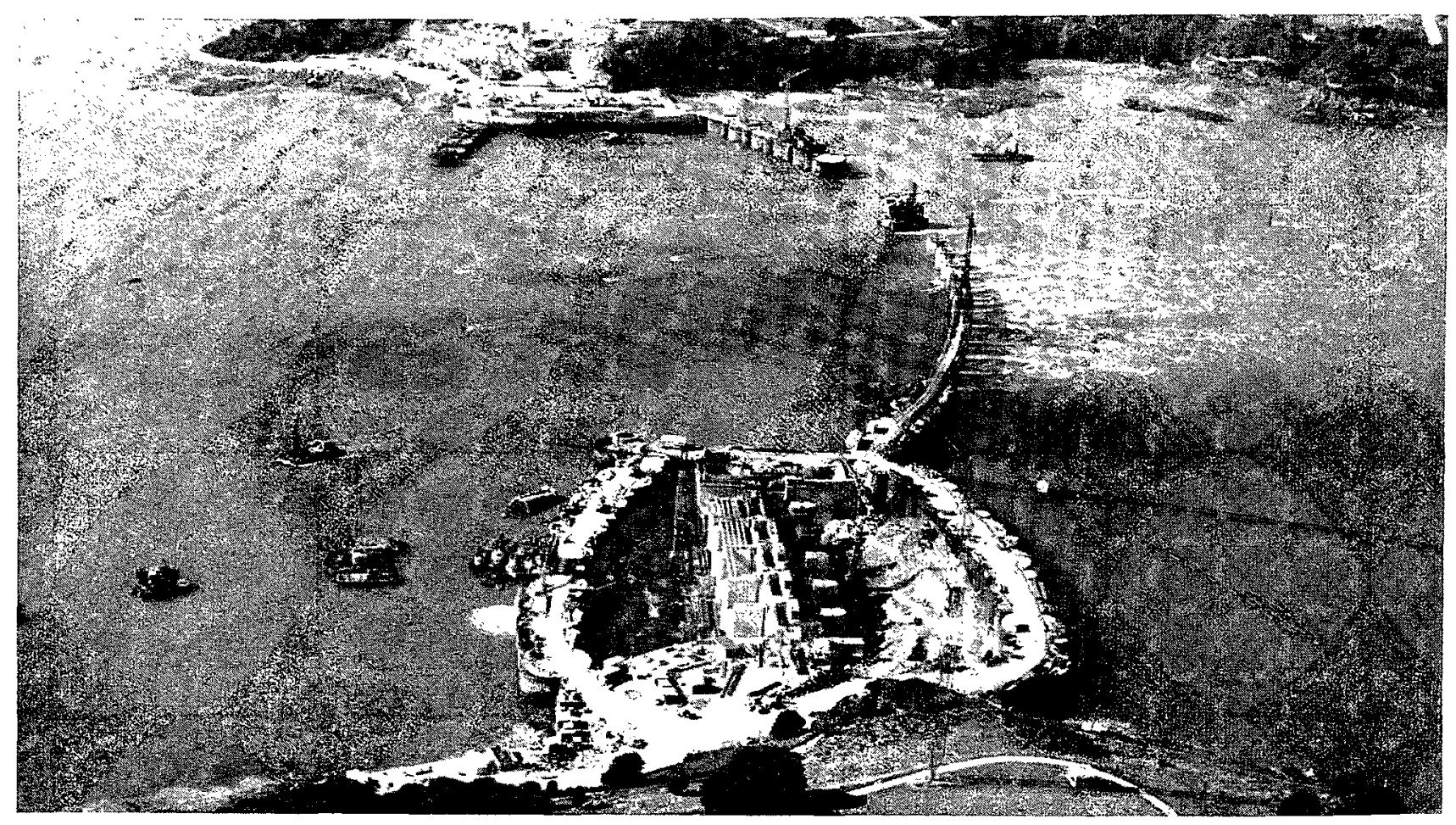

15 octobre 1962 : Le barroge mobile est en cours d'achèvement.

15th Getober 1962: Final work in progress on the dam spillway section

28 janvier 1963 : La partie Sud de l'enceinte des pertuis est en eau. Les travaux à l'air comprimé sont terminés. II reste un caisson à mettre en place.

28th January 1963: Southern part of spillway sluice area under water.

Compressed air work has been completed, leaving only the last caisson to be positioned.

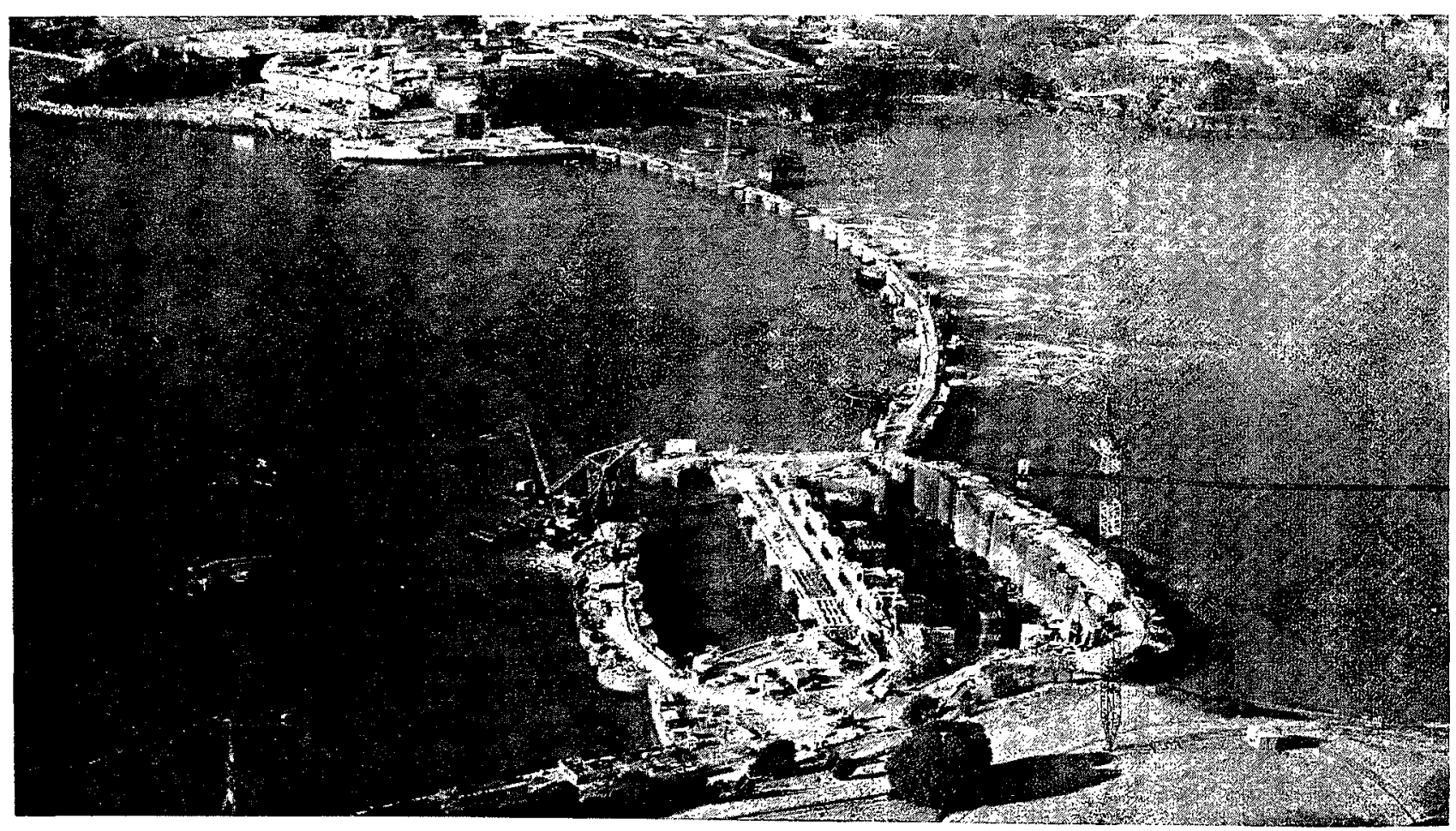




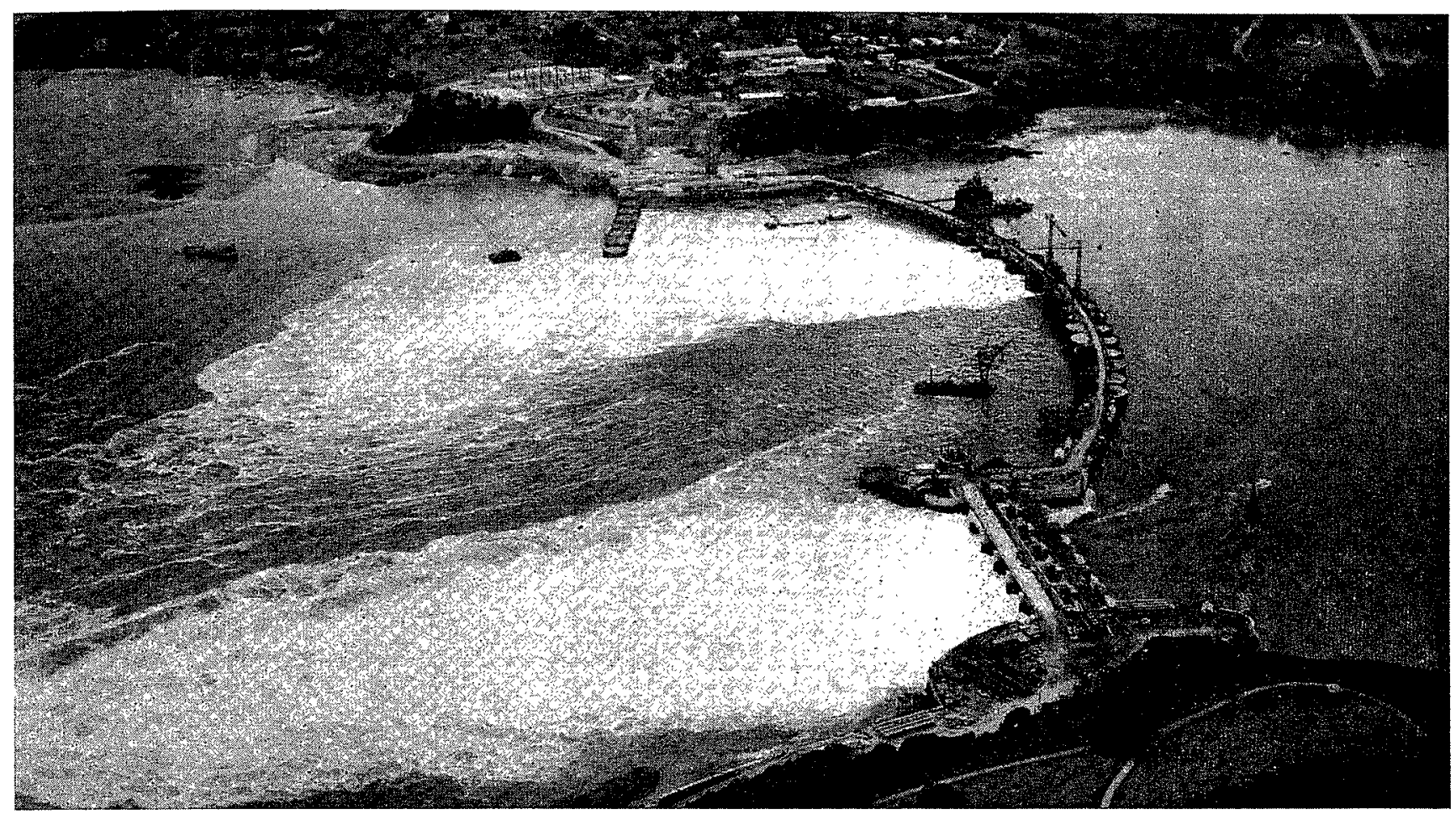

22 juin 1963 : Les six vannes sont ouvertes pour laisser passer le flot (marée de coefficient 95). II reste trois brèches à fermer.

22nd June 1963: Flood tide through the dam, with all six gates open (Tide coefficient 95). Three openings still remain to be closed.

7 juillet 1963 : Les deux dernières brèches viennent d'être fermées. Le remplissage et la vidange de l'estuaire s'opèrent uniquement par les six yannes (marée de coefficient 79).

7th July 1963: The last two openings have just been closed, leaving the six sluice gates to handle the full estuary inflow and outflow. (Tide ccefficient 79.)

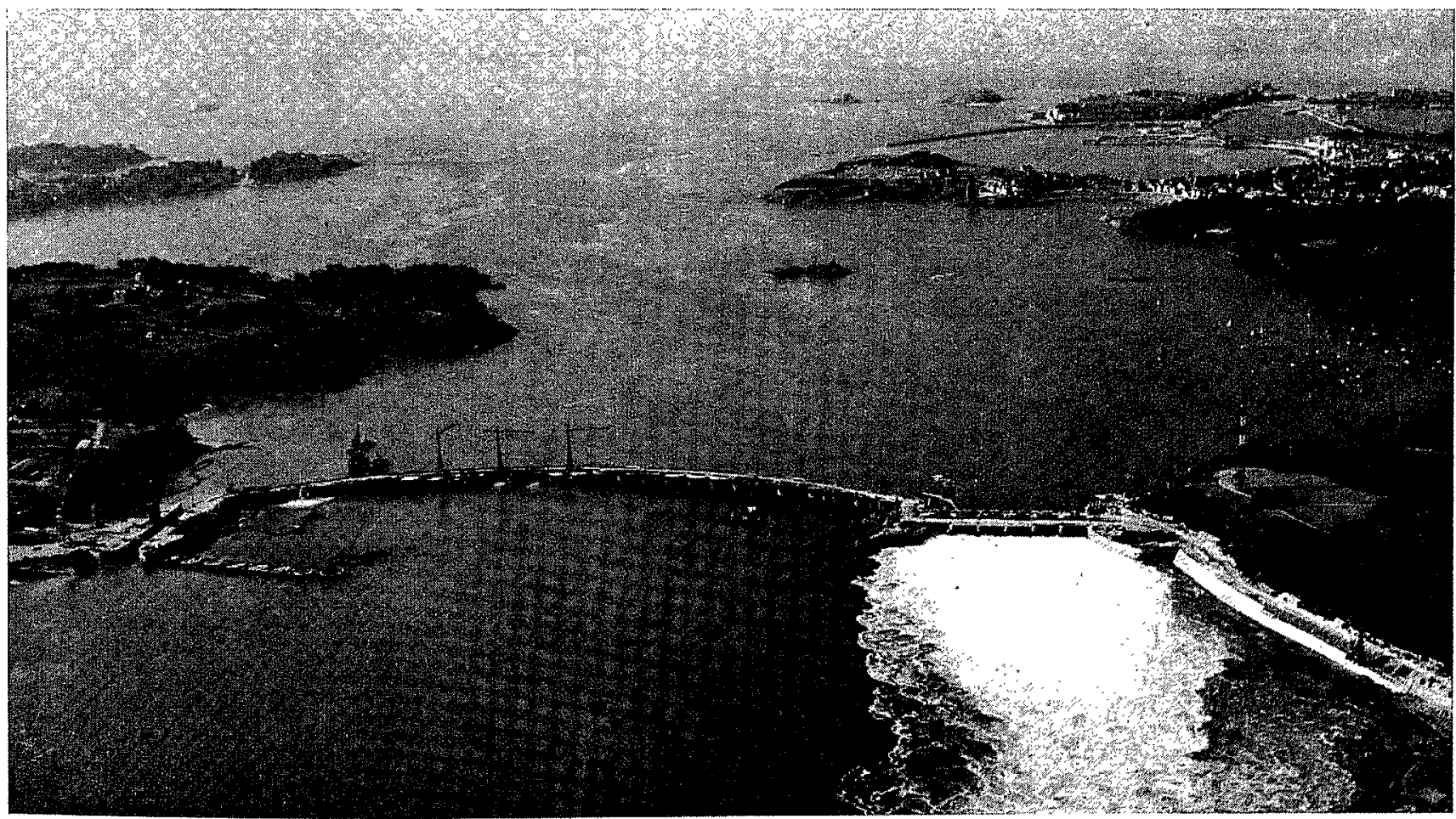




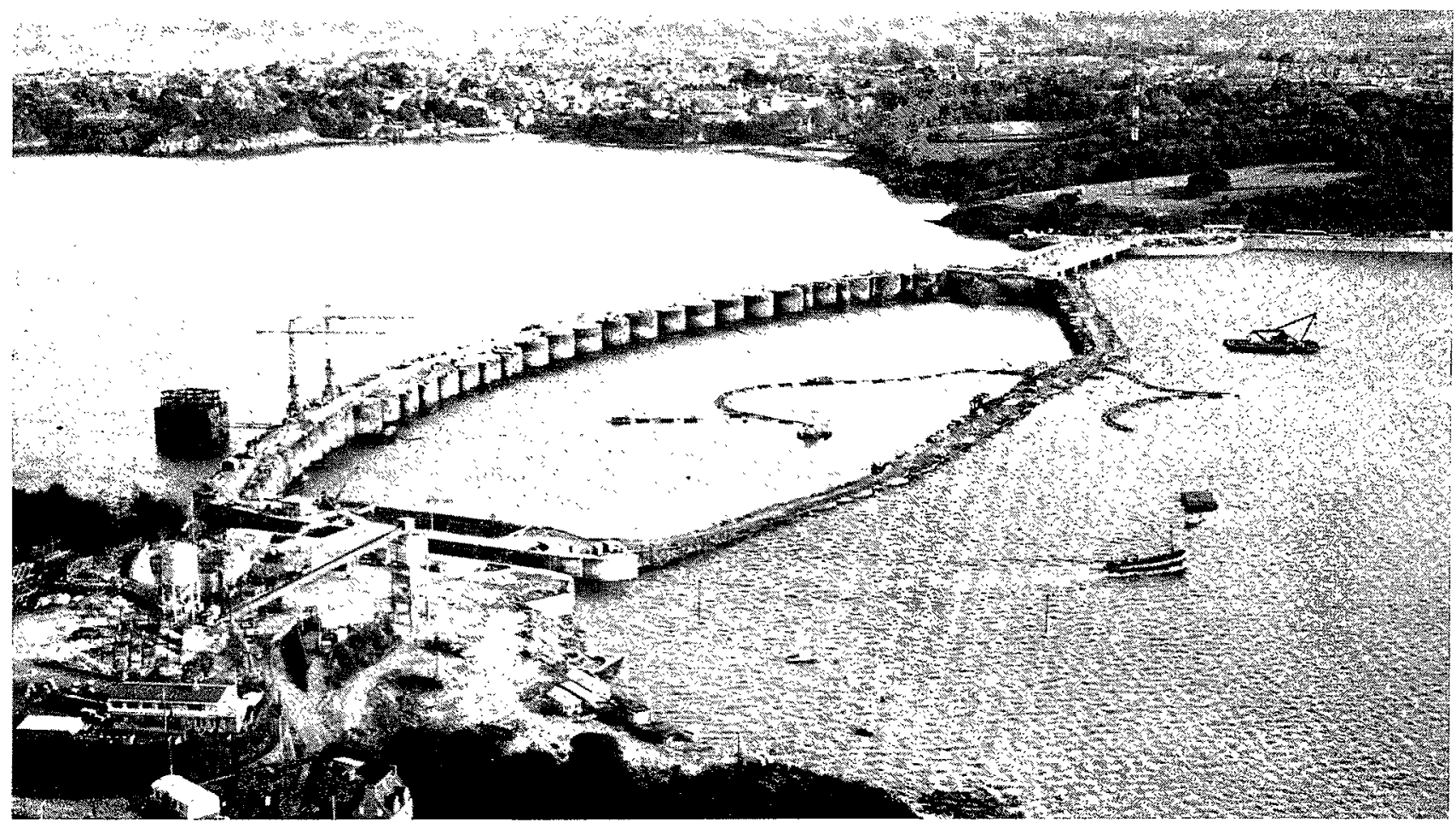

4 novembre 1963 : Le batordeau Sud est achevé. L'enceinte est en début d'épuisement (cote +3 environ).

4th November 1963: The south cofferdam in its final condition.

Pumping operations have just started behind it (water level about $3 \mathrm{~m}$ above datum).

26 novembre 1963 : L'épuisement de l'enceinte continue. La cote -9 est atteinte.

26th November 1963: Pumping operations at a more advanced stage (water level down to $9 \mathrm{~m}$ below datum).

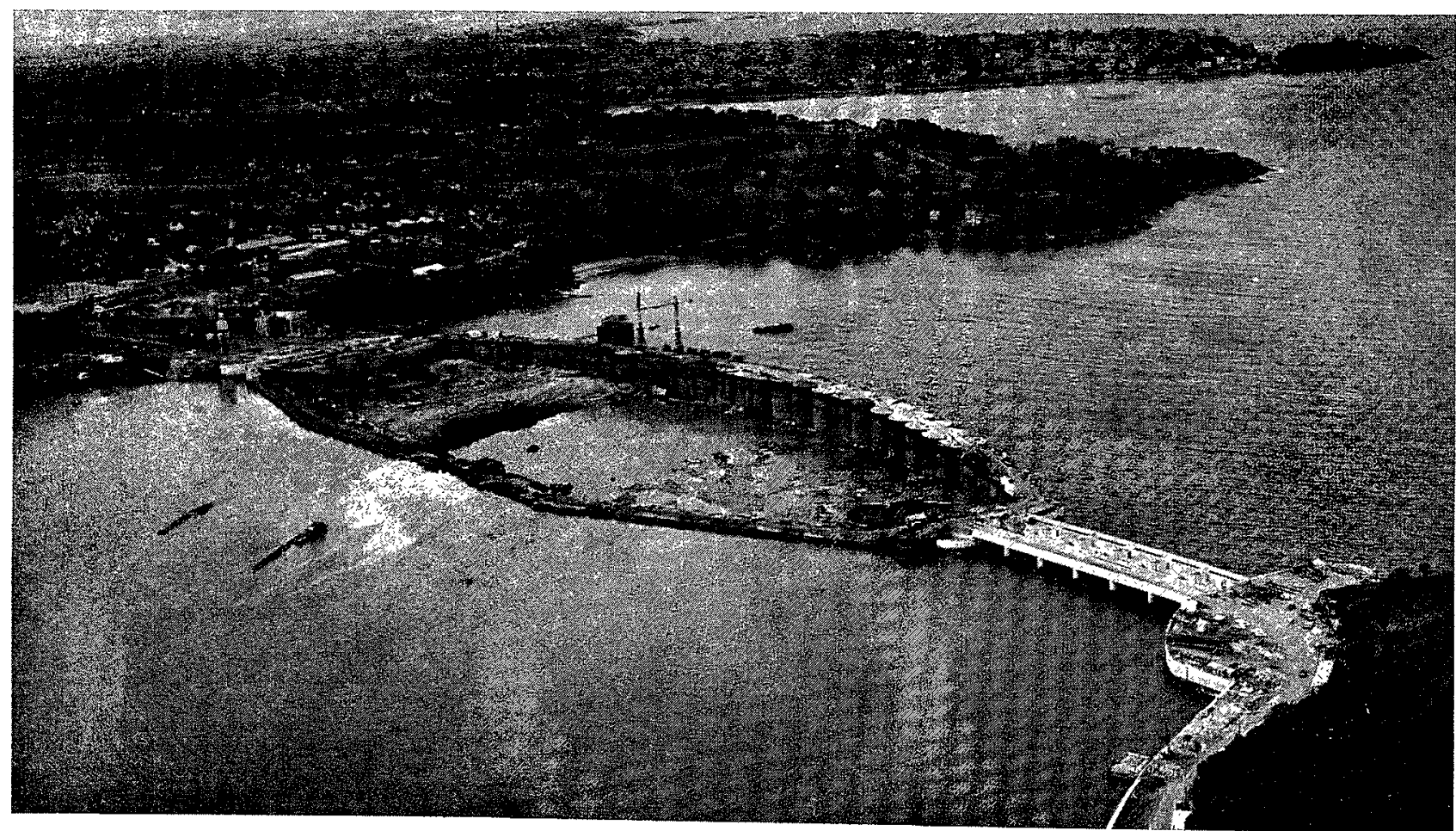

Photographies DouceT - Dimard. 